\title{
Synthesis and Characterization of Gellan Gum-Pyridoxine Nanoparticle for Treatment of Localized Peripheral Neuropathy
}

\author{
Vigneshwaran R, Sankar M, Vigneshkumar S.
}

\begin{abstract}
Biopolymer has full application as a drug carrier and scaffold in tissue engineering because of its biodegradability and non-toxicity. The present study is focused on novel biopolymer gellan gum as a drug delivery agent. The objective of the study is to synthesize the gellan gum nanoparticle by solvent evaporation emulsification method and characterize it using SEM, DLS, FTIR, and XRD. As pyridoxine is effectively used to treat diabetic peripheral neuropathy, it has been encapsulated with gellan gum and characterized. The antioxidant assay was performed using ABTS reagent, and the activity increases with increasing concentration of nanoparticle. Also, it has significant antimicrobial activity against gram-positive bacteria. Apart from this, the drug release kinetics of the gellan gum-pyridoxine nanoparticle was studied.
\end{abstract}

Keywords : Gellan gum, Pyridoxine, Drug carrier, Antioxidant

\section{INTRODUCTION}

Neuropathy is the condition that results from the damage or dysfunction of the nervous system - the communication network system that communicates information within the central nervous system and various parts present within the body. Several factors might induce the cause for peripheral neuropathy, which includes diabetes, vitamin deficiency, isoniazid therapy, tissue injury, alcohol abuse, and hormonal imbalance. Diabetes, a chronic metabolic disease characterized by abnormal levels of blood glucose due to insufficient secretion or abnormal function of insulin, is the leading factor of neuropathy. It has become the most significant global health-care problem of the $21^{\text {st }}$ century. There are 425 million peoples with diabetes, and at least $50 \%$ of them develop neuropathy within 25 years of diagnosis. Prolong abnormal glucose level, or unhealthy management of peripheral neuropathy can lead to limb amputation, a condition which causes serious lifestyle changes.

Diabetic peripheral neuropathy is the primary cause of limb amputation because of progressive loss of nerve function or nerve damage. So, it is hard for the nerves to carry the message to the brain, which results in numbness or painful tingling in affected parts. In the current medication, there is no complete cure or regeneration of the nerve. Symptomatic management is the only way to reduce neuropathic pain. Oxidative stress, axon, and myelin degradation in nerve cell is the primary cause of diabetic peripheral neuropathy.

Revised Manuscript Received on December 16, 2019.

Vigneshwaran $\mathbf{R}^{*}$, Department of Biotechnology, Kalasalingam Academy of Research and Education, Krishnankoil-626126 Email: vigneshwaran@klu.ac.in

Sankar M*, Department of Biotechnology, Mepco Schlenk Engineering College, Sivakasi 626005 Email: sankarmalayandi@gmail.com

Vigneshkumar S, Department of Biotechnology, Mepco Schlenk Engineering College, Sivakasi 626005 Email: viyadharshan@ gmail.com
Drug delivery has the potential to have a tremendous impact on the treatment of disease. However, a system has a wide variety of problems, such as less bioavailability, toxicity, and low targeting efficiency. Biopolymer-based drug carrier is advantageous over another polymer because of very high loading efficiency, controlled drug release for an extended period, and non-toxicity.

Transdermal drug delivery has defined as the non- invasive and patient compliance delivery of drug through the dermal layers. Bioavailability had improved by avoiding the metabolism take place in the liver, stomach, and intestine (Bajaj and Whiteman, 2011). Nanogel and nano emulsion are potent drug carrier formulation because of hydrophilicity. Also, their nanoscale structure enhances the drug concentration in the dermal layer.

The increasing trend for new drug carrier with improved properties, at the same time, ensuring simple, rapid, and economical manufacturing processes has motivated the pharmaceutical industry to search for the versatile compound. Biopolymers are one of the most multifunctional compounds employed in the food and pharmaceutical industry. Biopolymer produced by plant or microorganism is advantageous over synthetic polymer because of degradable and non-toxic nature. Biopolymers have a formal structure of a long chain of uniformly distributed molecules comparing with synthetic polymers.

Biopolymer is polymeric biomolecule which contains monomeric unit that is covalently bonded to form a largemolecule. It is classified into protein, lipid, and polysaccharides. Polysaccharide polymer like starch, chitin, chitosan, and cellulose are widely used as a drug carrier, wound dressing material, and scaffold in tissue engineering.

Gellan gum is considered as a bacterial polysaccharide produced by the bacterium Sphingomonas elodea. It is an aerobic fermentative product and used as an emulsifier in food additives. It has four repeating unit carbohydrates which include one rhamnose, two glucose, and one glucuronate. The natural form of gellan gum is called as high acyl gellan gum because it has two acyl substituents, i.e., acetate and glycerate. Both the acyl group is removed by alkali treatment. Hence it is called as low acyl gellan gum, which is commercially available in powdered form.

It is highly used in pharmaceutical and biomedical research such as gene therapy, bone, and tissue regeneration, wound dressing material, and bioadhesive drug formulation. 
Pyridoxine is also called as vitamin $\mathrm{B}_{6}$, which is a nutritional supplement. It is a derivative of pyridine and has the structure 2-methyl-3-hydroxy- 4,5-di (hydroxymethyl) pyridine (Urbanski, 1949). It is a water-soluble vitamin used to treat malnutrition, isoniazid therapy, and neuropathy. It acts as a cofactor for hundreds of biological reactions, which includes, synthesis of the neurotransmitter, myelin formation, and development of neuronal cell (Qu and Wu, 2004). It is also an excellent antioxidant and thus delays aging. Its deficiency is commonly seen in alcoholic individual, pregnant or lactating women. The RDA for pyridoxine is $1.3 \mathrm{mg}$ daily with an upper limit of $100 \mathrm{mg}$ daily (Hammond, 2014). The main objective for this present study was to synthesize gellan gum nanoparticles, characterize its physiochemical features, and estimate its antioxidant activity.

\section{MATERIALS AND METHODS}

\subsection{Preparation of Nanoparticles}

\subsubsection{Evaporation and Emulsification}

$0.05 \%$ of Gellan gum was dissolved in $1 \%$ acetic acid. $3 \mathrm{ml}$ this solution was added into $25 \mathrm{ml}$ acetone under constant stirring at $10,000 \mathrm{rpm}$ in a dropwise manner. Then $1 \mathrm{ml}$ of $5 \%$ Calcium chloride was added under constant stirring. After one hour of incubation, the sample was centrifuged. The pellet was lyophilized to obtain the pure powder form, and the supernatant was discarded.

\subsection{Physicochemical characterization}

\subsubsection{Hydrodynamic particle size analysis}

The mean values of hydrodynamic diameter and the relative polydispersity index (PDI) were determined by Dynamic light scattering (DLS) using Malvern zeta sizer ZS (Malvern Instruments, Malvern, UK). The weight of the freeze-dried nanoparticles was measured. $10 \mathrm{mg}$ of nanoparticles was taken and dissolved in $1 \mathrm{ml}$ of distilled water. The solution was taken in the cuvette, and hydrodynamic diameter was measured three times each at 20 runs.

\subsubsection{Zeta Potential}

The zeta potential values of the nanoparticles were assessed by determining electrophoretic mobility with the Malvern ZS equipment. The nanoparticle solution was taken in the zeta potential cuvette. The zeta potential of the nanoparticle was measured two times each at 50 runs.

\subsubsection{FTIR Analysis}

FT-IR spectroscopy is a powerful technique used for studying the physicochemical and conformational properties of polysaccharides. Synthesis composite was analyzed in the FTIR instrument, within the frequency range of 500 to 5000 $\mathrm{cm}^{-1}$, to know the presence of different functional groups. The bands in the range of $4000-1500 \mathrm{~cm}^{-1}$ are typically due to functional groups such $-\mathrm{OH}, \mathrm{C}=\mathrm{O}$, and $\mathrm{CH}_{3}$ (Silver and Braga,2012). The rangefrom $1500-400 \mathrm{~cm}^{-1}$ is referred to as the fingerprint region and generally caused by intra-molecular phenomenon peculiar to each material. The sample was dispersed in water and analyzed with FTIR instrument.

\subsubsection{Scanning Electron Microscopy}

The morphology analysis of the prepared composite was performed by Scanning Electron Microscopy. In Scanning Electron Microscope, high-energy electrons are used to generate many signals at the surface of solid specimens. The derived signals from electron-sample interactions give

information about the samples external morphology (texture), chemical composition, and crystalline structure.

2.2.5 X-ray Diffraction Analysis

$\mathrm{XRD}$ is a rapid analytical tool used for the phase identification of crystalline material. X-ray diffraction studies analyzed the phased nature of the synthesized composite. XRD is based on constructive interference of monochromatic X-rays and the crystalline samples.

\subsection{Application studies}

\subsubsection{Antioxidant activity}

ABTS (2, 2' azino-bis (3-ethlbenzthiazoline-6-sulphonic acid)) ABTS assay was performed to check the total antioxidant capacity of the sample. The stock solution was prepared by adding $19.5 \mathrm{mg}$ ABTS and $3.3 \mathrm{mg}$ potassium persulfate in $7 \mathrm{ml}$ of distilled water. The stock reagent was kept at dark for 12 hours. The useful ABTS reagent was prepared by adding $1 \mathrm{ml}$ stock reagent to $60 \mathrm{ml}$ of methanol.

\subsubsection{Antimicrobial activity}

The antimicrobial activity of the complex was done by the disc diffusion method (Dutta, 2009). $100 \mu \mathrm{l}$ of bacterial suspension was spread in the agar medium using L-rod, and the sample disc was dipped into the sample. The disc was dried and placed on the plates, which were incubated at $37^{\circ} \mathrm{C}$ for overnight incubation. Inhibition zones were measured based on the average diameter of the bright area, directly on the plate (Ciprari, 2004)

\subsubsection{Drug Release Study}

The release of Pyridoxine from the Gellan gum pyridoxine nanoparticles was studied using simulated fluids. SBF (Kokubo et al 1990), SGF, SGF with pepsin (Fu et al., 2002) and SIF (Stippler et al 2004) was prepared and used as simulated fluids. Gellan gum of various concentrations was used as standard. After an incubation period of 1 hour, the spectrophotometric analysis was done at $292 \mathrm{~nm}$.

\section{RESULTS AND DISCUSSION}

\subsection{Average Size Distribution Analysis}

Particle size analyzer (MALVERN) was used to measure the approximate size of the synthesized particle. The standard size distribution of gellan gum was shown in Fig 3.1(a), in which particle had size distribution of range 1 to $5 \mathrm{~nm}, 20$ to $30 \mathrm{~nm}$ and 200 to $400 \mathrm{~nm}$ with a peak intensity of $8.1 \%$, $25.8 \%$, and $66.2 \%$ respectively. Thus, the particles are in the different size. The standard size distribution of pyridoxine encapsulated gellan gum particle was shown in Fig 3.1(b). This particles size was also heterogeneous, and the range of size was found to be 2 to $3 \mathrm{~nm}, 100$ to $200 \mathrm{~nm}$, and 400 to 900 $\mathrm{nm}$.

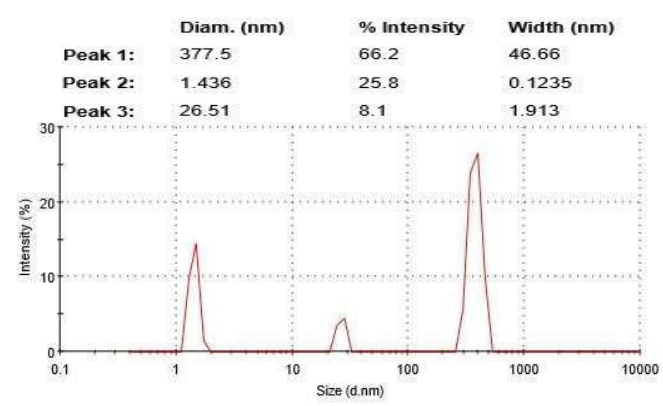




$\begin{array}{llll} & \text { Diam. (nm) } & \text { \% Intensity } & \text { Width }(\mathrm{nm}) \\ \text { Peak 1: } & 585.7 & 70.4 & 112.4 \\ \text { Peak 2: } & 2.550 & 19.4 & 0.2697 \\ \text { Peak 3: } & 122.7 & 10.2 & 14.35\end{array}$

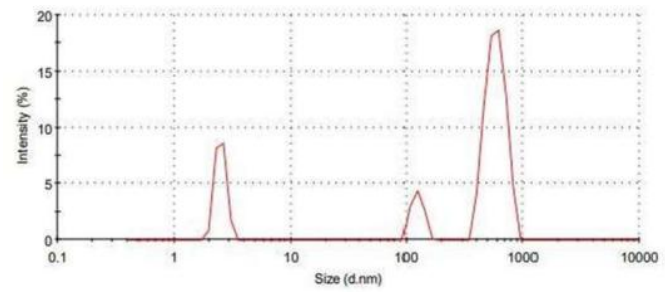

Fig 3.1(a) Size distribution of particle (b) Size distribution of Pyridoxine encapsulated gellan gum particle

\subsection{Zeta Potential}

Particle colloidal stability and the surface charge was analyzed by zeta potential data shown in Fig 3.2(a) and Fig 3.2(b).
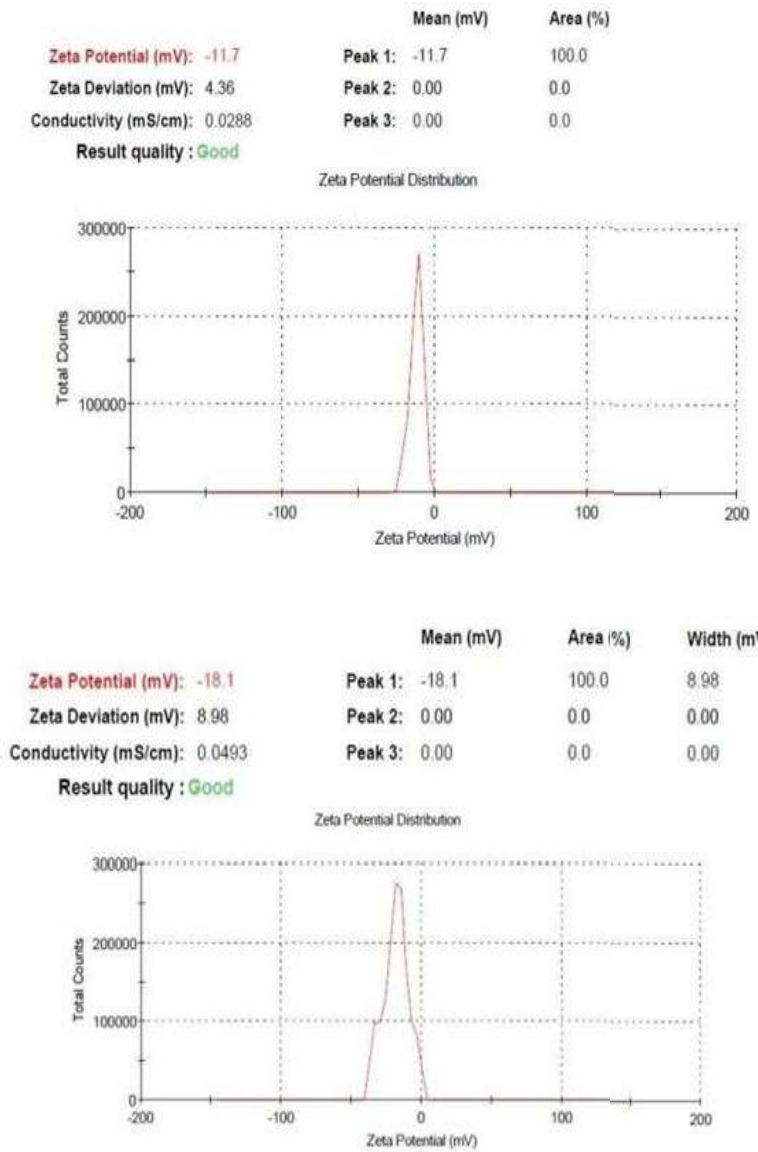

Fig 3.2(a) Zeta potential of gellan gum particle (b) Zeta potential of pyridoxine encapsulated gellan gum.

\subsection{FTIR Analysis}

The individual IR spectrum of Gellan gum, Pyridoxine, Gellan gum-Pyridoxine and the concrete mixture was shown in the fig $3.3 \mathrm{a}, \mathrm{b}, \mathrm{c}$ and $\mathrm{d}$. The broadband at $3128 \mathrm{~cm}^{-1}$ originates from the stretching of $\mathrm{C}-\mathrm{H}$ group on gellan gum. The intensity of this band decreases in pyridoxine encapsulated gellan gum particle, and formation of $3480 \mathrm{~cm}^{-1}$ peaks corresponds to $\mathrm{O}-\mathrm{H}$ stretch were seen in Fig 3.3 (c), since $\mathrm{O}-\mathrm{H}$ group was predominantly present in pyridoxine, which confirms the binding of pyridoxine to gellan gum. Five of six ring stretching modes of the pyridine ring of pyridoxine appear in the region 1650-1200 $\mathrm{cm}^{-1}$ (Srivastava and Rani, 2014).
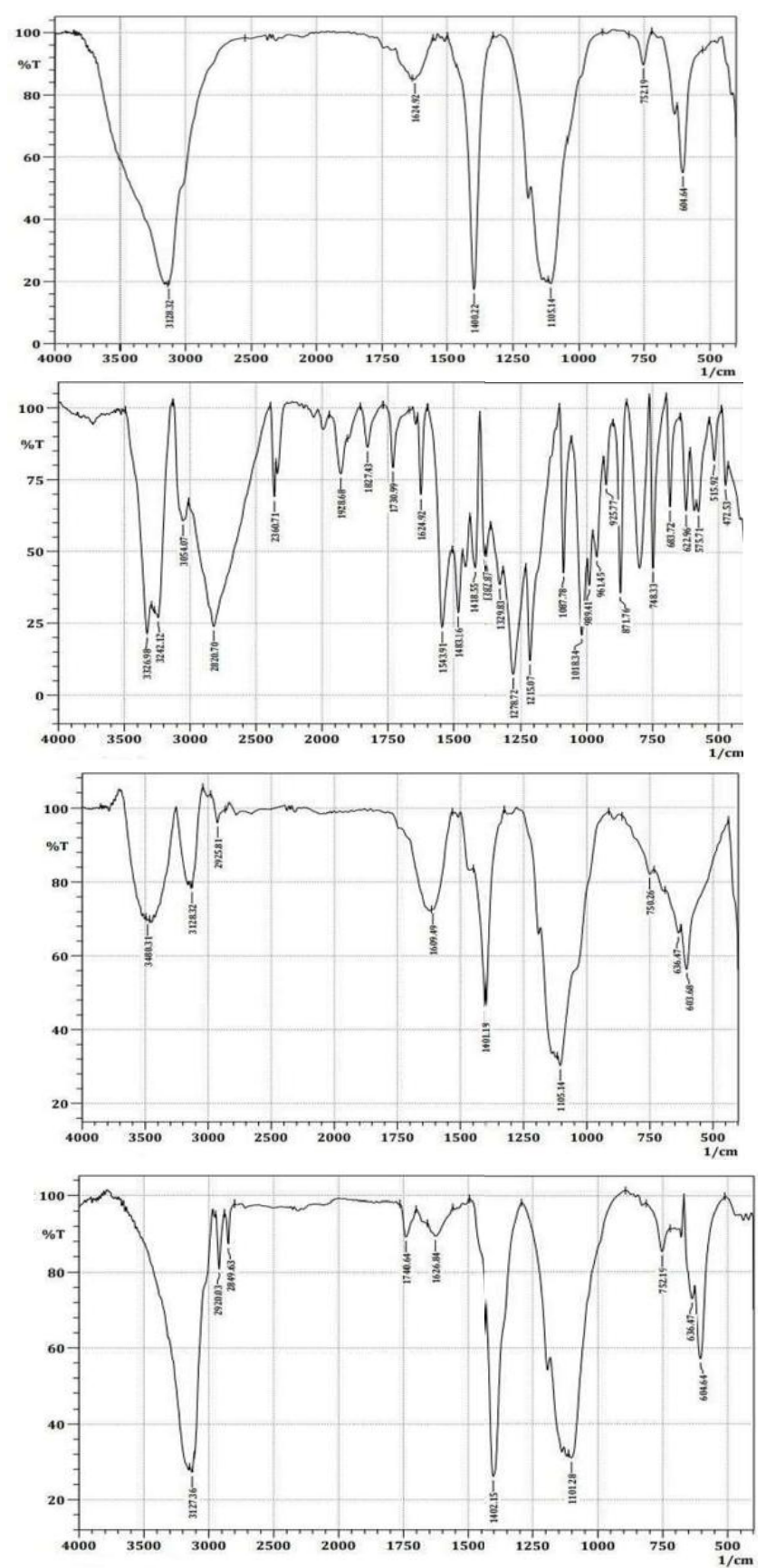

Fig 3.3 FTIR spectrum indicating (a) Gellan gum nanoparticle (b) Pyridoxine (c) Pyridoxine encapsulated Gellan gum nano- particle (d) Physical mixture of gellan gum and pyridoxine.

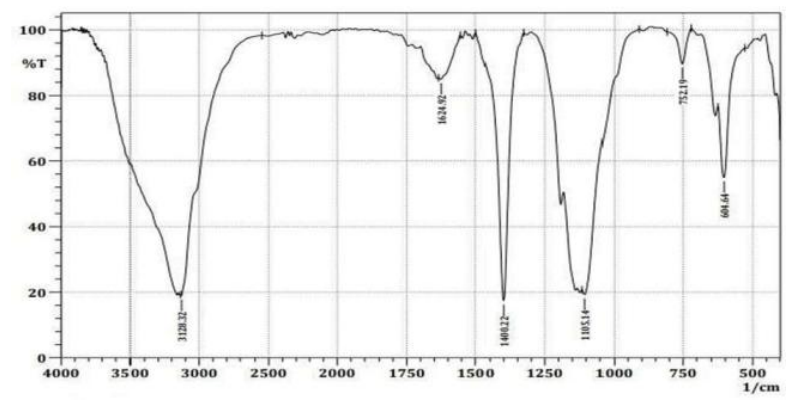

\subsection{X-Ray Diffraction Analysis}

The XRD pattern of Gellan gum, Pyridoxine, and synthesized Gellan gum-Pyridoxine were shown in Fig 3.4(a), (b) and (c). The XRD pattern of gellan gum (Fig 3.4(a)) shows broad peaks, which indicates that gellan is

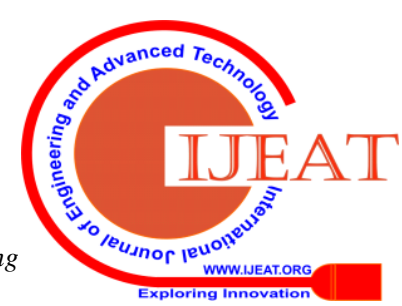


semi-crystalline and amorphous.

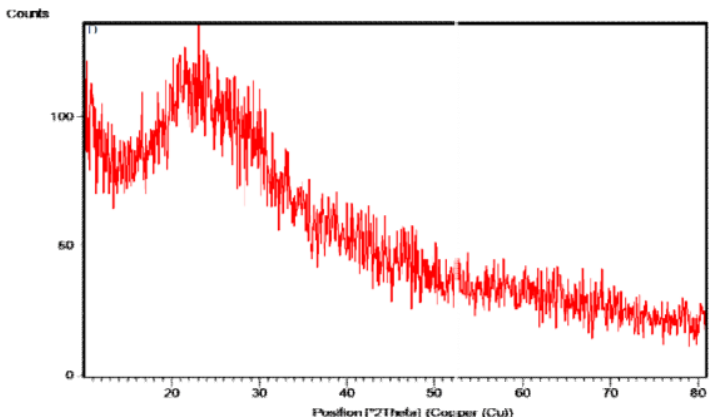

The diffraction pattern of pyridoxine (Fig 3.4(b)) shows a sharp peak, which indicates that pyridoxine is highly crystalline. Gellan gum-Pyridoxine complex XRD pattern also shows broad peaks, which indicates the complex is semi-crystalline.
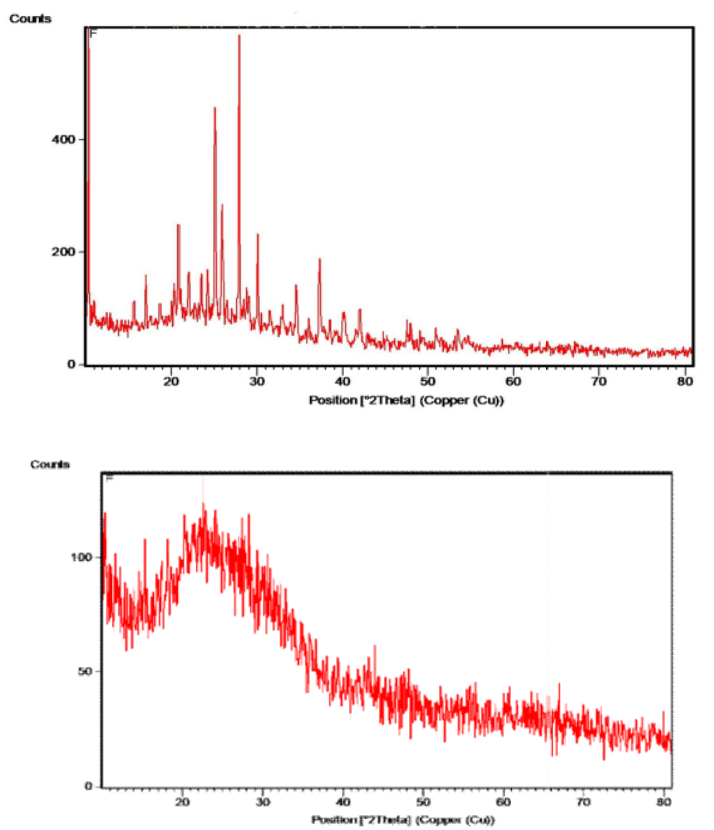

Fig 3.4 XRD pattern indicating (a) gellan gum (b) Pyridoxine Gellan gum-Pyridoxine

\subsection{Scanning Electron Microscope Analysis}

Morphology of the composite was analyzed by Scanning Electron Microscope (SEM). SEM analysis shows the aggregation of a particle with one another with an irregular shape. The aggregated particle has a size range of $700 \mathrm{~nm}$ to $10 \mu \mathrm{m}$. The SEManalysis was contrary to the DLS because of particle aggregation.
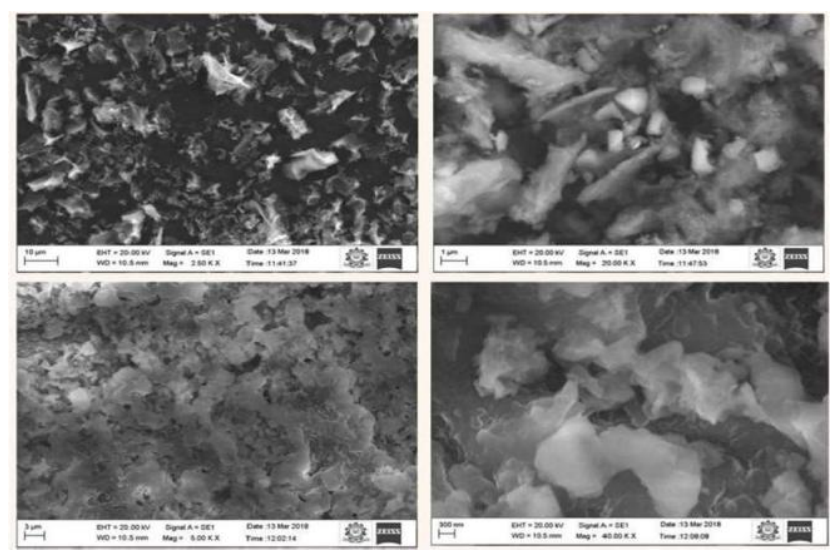

\section{Antioxidant Assay}

ABTS scavenging assay was performed to estimate the antioxidant activity of the compounds. This assay was based on the ability of ABTS, stable free radicals, to be quenched and thereby decolorize in the presence of antioxidants resulting in a reduction in absorbance values. The results showed that antioxidant activity of the gellan gum, pyridoxine, and complex was increased with increasing concentration. At $5 \mathrm{mg} / \mathrm{ml}$ concentration, both pyridoxine and complex have an antioxidant activity like the positive control (Ascorbic acid).

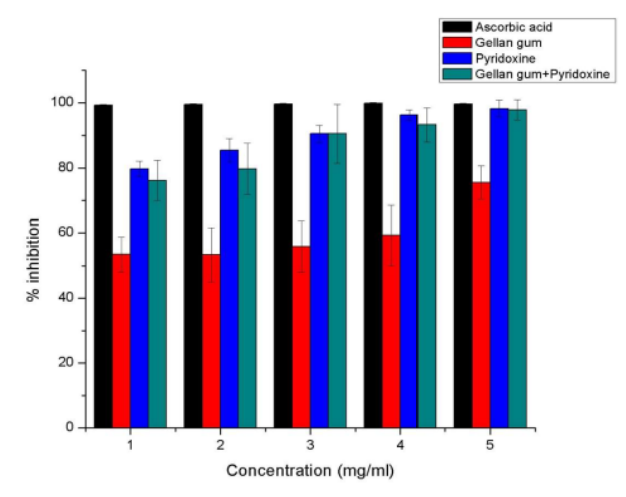

\section{Drug Release Kinetics}

Pyridoxine release at various physiologically simulated fluid was studied (Fig 3.6). It was observed that pyridoxine release was constant for simulated gastric fluid with and without pepsin. Whereas increases in pyridoxine release were observed in simulated intestinal fluid.

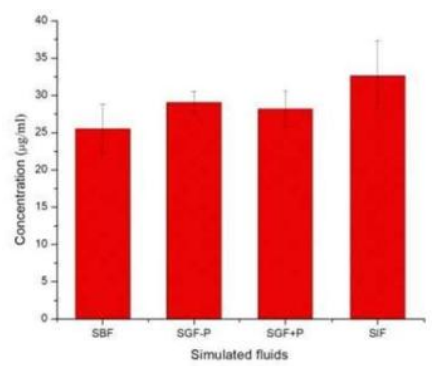

Fig 3.6 Pyridoxine release at simulated fluids.

\section{Antimicrobial Activity}

Antimicrobial activity of the compounds was evaluated by disc diffusion method. The complex, gellan gum and pyridoxine were tested against gram positive and gramnegative bacteria, using gentamicin as control (Fig 3.7). In this study, the zone of inhibition was observed for gellan gum (GG) and gellan gum- pyridoxine complex (GP) for Bacillus subtilis. Whereas no zone of inhibition was observed for Pseudomonas putida. Hence, the complex particle has antimicrobial activity against gram-positive bacteria (B. subtilis) at a concentration of $2 \mathrm{mg} / \mathrm{ml}$. 

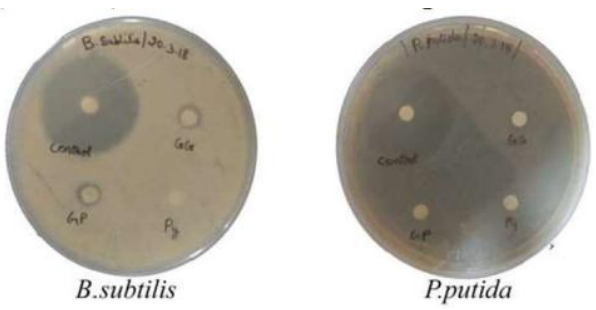

Fig 3.7 Antimicrobial activity of individual component and complex.

\section{CONCLUSION}

In this study, the gellan gum particles were prepared by solvent evaporation- emulsification method. The particle size range varies from nano to micro scale was determined by dynamic light scattering analysis. Scanning electron microscopy image revealed microparticles was due to the aggregation of particle. XRD analysis shows the semi-crystalline nature of the particle. Using FTIR function group modification and pyridoxine encapsulation was confirmed. The particles can be uptake or interact by the cell because of negative zeta potential. The complex particle has good antioxidant activity and antimicrobial activity against gram- positive bacteria $B$. subtilis. In vitro drug release kinetics also shows the efficiency of drug release and particle degradation.

\section{REFERENCES}

1. Bajaj, S. and A. Whiteman (2011) "Transdermal drug delivery in pain management." Continuing Education in Anaesthesia, Critical Care \& Pain: mkq054.

2. Ciprari, D. L. (2004). "Mechanical characterization of polymer nanocomposites and the role of interphase, Georgia Institute of Technology."

3. Bakker, K. and W. H. van Houtum. (2005). "The International Diabetes Federation focuses on the diabetic foot." Current diabetes reports 5(6): 436-440.

4. Dutta, P. K. and J. Dutta. (2004). "Chitin and chitosan: Chemistry, properties and applications."

5. Ebringerová, A. (2005). "Structural diversity and application potential of hemicelluloses. Macromolecular Symposia, Wiley Online Library."

6. Ebringerova, A. and T. Heinze (2000). "Xylan and xylan derivativesbiopolymers with valuable properties, Naturally occurring xylans structures, isolation procedures and properties." Macromolecular rapid communications 21(9): 542-556.

7. Felt, O. and P. Buri. (1998). "Chitosan: a unique polysaccharide for drug delivery." Drug development and industrial pharmacy 24(11): 979-993.

8. Fowler, M. J. (2008). "Microvascular and macrovascular complications of diabetes." Clinical diabetes 26(2): 77-82.

9. Galer, B. S. and A. Gianas. (2000). "Painful diabetic polyneuropathy: epidemiology, pain description, and quality of life." Diabetes research and clinical practice 47(2): 123- 128.

10. Garcia, R. B. and T. Nagashima Jr. (2001). "Preparation of micro and nanoparticles from corn cobs xylan." Polymer Bulletin 46(5): 371-379.

11. Gerster, H. (1996). "The importance of vitamin B 6 for development of the infant. Human medical and animal experiment studies." Zeitschrift fur Ernahrungswissenschaft 35(4): 309-317.

12. Hachem, K. and C. Faugeron. (2016). "Structural Investigation of Cell Wall Xylan Polysaccharides from the Leaves of Algerian Argania spinosa." Molecules 21(11): 1587.

13. Jayakumar, R. and M. Prabaharan. (2010). "Novel chitin and chitosan nanofibers in biomedical applications." Biotechnology advances 28(1): 142-150.

14. Kapur, S. and R. J. McKendry (2005). "Treatment and outcomes of diabetic muscle infarction." JCR: Journal of Clinical Rheumatology 11(1): 8-12.
15. Khalil, H. and H. Chambers. (2016). "Vitamin B for treating diabetic peripheral neuropathy." The Cochrane Library

16. Kumar, S. and Y. S. Negi (2012). "Nanoparticles Synthesis from Corn Cob (Xylan) and Their Potential Application as Colon-Specific Drug Carrier." Macromolecular Symposia, Wiley Online Library.

17. Kumar, S. and Y. S. Negi (2014). "Cellulose and xylan based prodrug of diclofenac sodium: synthesis, physicochemical characterization and in vitro release." International Journal of Polymeric Materials and Polymeric Biomaterials 63(6): 283- 292.

18. Kumari, A. and S. K. Yadav. (2010). "Biodegradable polymeric nanoparticles based drug delivery systems." Colloids and Surfaces B: Biointerfaces 75(1): 1-18.

19. Liu, H. and Y. Fu. (2016). "A Fibrous Localized Drug Delivery Platform with NIR- Triggered and Optically Monitored Drug Release." Langmuir: the ACS journal of surfaces and colloids 32(35): 9083.

20. Martyn, C. and R. Hughes (1997). "Epidemiology of peripheral neuropathy." Journal of Neurology, Neurosurgery \& Psychiatry 62(4): 310-318.

21. Mehdizadeh, T. and H. Tajik. (2012). "Antibacterial, antioxidant and optical properties of edible starch-chitosan composite film containing Thymus kotschyanus essential oil." Veterinary Research Forum, Veterinary Research Forum.

22. Melo-Silveira, R. F. and G. P. Fidelis. (2011). "In vitro antioxidant, anticoagulant and antimicrobial activity and in inhibition of cancer cell proliferation by xylan extracted from corn cobs." International journal of molecular sciences 13(1): 409- 426.

23. Oliveira, E. E. and A. E. Silva. (2010). "Xylan from corn cobs, a promising polymer for drug delivery: Production and characterization." Bioresource technology 101(14): 5402- 5406.

24. Pilowsky, I. and I. Crettenden. (1985). "Sleep disturbance in pain clinic patients." Pain 23(1): 27-33.

25. Qu, W. and K. Wu. (2004). "Voltammetric determination of pyridoxine (Vitamin B by use of a chemically-modified glassy carbon electrode." Journal of pharmaceutical and biomedical analysis 36(3): 631-635.

26. Srivastava, M. and P. Rani. (2014). "Experimental and theoretical studies of vibrational spectrum and molecular structure and related properties of pyridoxine (Vitamin B6)." Spectrochimica Acta Part A: Molecular and Biomolecular Spectroscopy 120: 274-286.

\section{AUTHORS PROFILE}

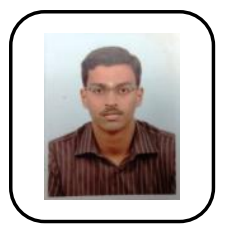

Vigneshwaran $\mathbf{R}$ has completed his $\mathrm{M}$. Tech. degree in the field of Biotechnology in the year of 2013 and submitted his PhD dissertation in 2019 in the field of molecular biology and genetic engineering. At present he is working as Assistant Professor at the Department of Biotechnology, Kalasalingam Academy of Research and Education, Krishnankoil-626126.

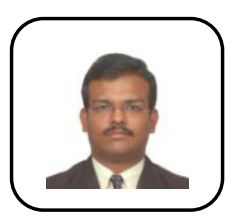

Sankar M has completed his M. Tech. degree in the field of Biotechnology in the year of 2009 and pursuing his $\mathrm{PhD}$ in the field of bioprocess engineering. At present he is working as Assistant Professor at the Department of Biotechnology, Mepco Schlenk Engineering College, Sivakasi-626005.

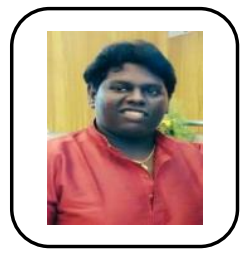

Vigneshkumar $\mathbf{S}$ has completed his $\mathbf{M}$. Tech. degree in the field of Biotechnology in the year of 2014 and submitted his $\mathrm{PhD}$ dissertation in the field of material science. At present he is working as a research scholar at the Department of Biotechnology, Mepco Schlenk Engineering College, Sivakasi-626005. 